\title{
MAPEAMENTO DAS ÁREAS DE CANA-DE-AÇÚCAR NA REGIÃO NORTE FLUMINENSE - RJ POR USO DE TÉCNICAS DE SENSORIAMENTO REMOTO
}

\author{
JOSÉ C. MENDONÇA ${ }^{1}$, RAMON M. DE FREITAS ${ }^{2}$, DANIEL A. DE AGUIAR ${ }^{3}$, \\ ELIAS F. DE SOUSA ${ }^{4}$, RODRIGO DE A. MUNIZ ${ }^{5}$, BARBARA DOS S. ESTEVES ${ }^{6}$
}

\begin{abstract}
RESUMO: Este trabalho teve por objetivo avaliar a dinâmica da ocupação do uso do solo cultivado com a cultura da cana-de-açúcar nos seis principais municípios produtores da região Norte Fluminense - RJ, entre o período de 1984 a 2007. Foram utilizadas 18 imagens do sensor TMLandsat, técnicas de interpretação visual e de Modelo Linear de Mistura Espectral (MLME), para gerar mapas temáticos das áreas ocupadas com a cultura. Com base nesses mapas, foi possível analisar a espacialização das áreas de cana e quantificá-las em cada município. Dos resultados obtidos, pode-se concluir que, entre os anos de 2004 a 2007, ocorreu um decremento total na área ocupada com a cultura, em 43.308,33 ha. A partir do ano de 2000 até 2007, houve um incremento de 24.422,72 ha, principalmente nos municípios de Campos dos Goytacazes, São Francisco de Itabapoana e Cardoso Moreira. Os resultados indicam que o uso do MLME permitiu um mapeamento mais exato das áreas ocupadas com cana-de-açúcar.
\end{abstract}

PALAVRAS-CHAVE: modelo linear de mistura espectral, uso do solo, Landsat.

\section{MAPPING OF SUGARCANE AREAS IN THE NORTH FLUMINENSE REGION, RJ USING REMOTE SENSING TECHNIQUES}

\begin{abstract}
This study aimed to evaluate sugarcane growth dynamics occupation in land cultivated at six major cities of the North Fluminense, Rio de Janeiro, from 1984 to 2007. Eighteen images of Landsat TM sensor, visual interpretation and linear spectral mixing model (MLME) were used to generate thematic maps of sugar cane plantation spatial distribution and quantification. Based on these maps was possible to analyze the sugarcane field spatial distribution quantifying them in each municipality. The results indicated that between the years 2004 to 2007, there was a decrement in the total area occupied by the culture in 43,308.33 ha and, from 2000 to 2007 and increase of 24,422.72 ha, mainly in the Campos dos Goytacazes, São Francisco de Itabapoana and Cardoso Moreira municipalities. The MLME used allowed a real live mapping of the sugarcane areas.
\end{abstract}

KEYWORDS: linear spectral mixture model, soil use, Landsat.

\section{INTRODUÇÃO}

A cana-de-açúcar é uma cultura de grande importância nacional, ocupando grandes extensões de terras, sendo o Brasil o maior produtor e exportador do mundo, seguido pela Índia e Austrália. De acordo com a CONAB (2009), o Estado do Rio de Janeiro é o $11^{\circ}$ produtor nacional da cultura, tendo produzido, na safra de 2008/2009, 3.558,3 toneladas, sendo a região Norte Fluminense a principal produtora.

A cana-de-açúcar vem recebendo cada vez mais destaque no cenário mundial por ser uma cultura de grande eficiência na produção de biocombustíveis e consequente mitigação da

\footnotetext{
${ }^{1}$ Eng ${ }^{\mathrm{o}}$ Agrônomo D.Sc. LAMET/UENF. Rod. Amaral Peixoto Km 163, Av Brenannd s/n - Imboassica. CEP 27925-310, Macaé, RJ. Fone (22) 27366501, mendonca@uenf.br.

${ }^{2}$ Doutorando em Computação Aplicada, INPE - São José dos Campos, SP. Fone (12) 39456000. Email: ramon@ dsr.inpe.br.

${ }^{3}$ Geógrafo, Doutorando em Sensoriamento Remoto, DSR/INPE. São José dos Campos, SP. Fone (12) 39456000, daniel@ dsr.inpe.br.

${ }^{4}$ Doutor em Produção Vegetal, Professor Titular, LEAG/UENF - Campos dos Goytacazes - RJ, efs@ @enf.br.

${ }^{5}$ Eng ${ }^{\mathrm{o}}$ Agrônomo, Mestre em Produção Vegetal, LEAG/UENF - Campos dos Goytacazes - RJ, uenf.rodrigo@ gmail.com.

${ }^{6}$ Eng ${ }^{\mathrm{a}}$ Agrônoma, Mestre em Produção Vegetal, LEAG/UENF - Campos dos Goytacazes - RJ, barbbarase@ gmail.com.

Recebido pelo Conselho Editorial em: 5-7-2010

Aprovado pelo Conselho Editorial em: 15-3-2011
} 
intensificação do efeito estufa. A cultura é uma das mais tecnificadas e capacitadas no que diz respeito ao uso de técnicas para seu gerenciamento (GOLDEMBERG \& GUARDABASSI, 2009).

O uso do sensoriamento remoto na agricultura está associado com a identificação e o mapeamento de culturas, entre outras aplicações possíveis. No Brasil, CHEN et al. (1982) realizaram um estudo comparativo da eficiência de diferentes classificadores automáticos na identificação e na estimativa da área ocupada pela cultura do trigo e ASSUNÇÃO \& DUARTE (1983) realizaram um dos principais esforços sistemáticos para a utilização de dados orbitais no mapeamento de cultivos.

Entretanto, BENEDIKTASSON et al. (1990) ressaltam que os procedimentos convencionais de classificação automática apresentam limitações decorrentes, principalmente, da resolução das imagens, da ocorrência de pixels misturados e da ambiguidade das classes, as quais, algumas vezes, podem resultar em mapeamentos incorretos.

SHIMABUKURO \& SMITH (1991) e SHIMABUKURO et al. (1998) demonstraram, através do uso de Modelos Lineares de Mistura Espectral (MLME), que as imagens de fração vegetação distinguem facilmente florestas tropicais densas e áreas desmatadas. A utilização de imagens de fração vegetação em ecossistemas florestais também foi demonstrada em estudos realizados nas florestas de coníferas norte-americanas através de SABOL et al. (2002).

A estimativa da produção de cana-de-açúcar é essencial no planejamento agrícola e na comercialização da produção gerada pelo setor. A variabilidade espacial da cultura é uma das causas principais de erros no processo de estimativa de produção. Nesse sentido, o sensoriamento remoto desponta como uma das ferramentas mais viáveis para mapear esta variabilidade e auxiliar no processo de estimativa de produção, possibilitando, por meio de processamento digital, gerar mapas que indiquem a variabilidade espacial da cultura (ABDEL-RAHMAN \& AHMED, 2008; AGUIAR et al., 2009; RUDORFF et al., 2010).

Desde o ano de 2003, o monitoramento do cultivo da cana-de-açúcar no Estado de São Paulo vem sendo realizado, com sucesso, pelo Instituto Nacional de Pesquisas Espaciais - INPE, através do projeto CANASAT (www.dsr.inpe.br/canasat). Em 2005, os demais estados produtores de cana-de-açúcar da região Centro-Sul também foram inseridos no projeto, exceto os Estados do Rio de Janeiro e Espírito Santo. Nesses estados, a identificação da cana-de-açúcar é dificultada pelo fato de o cultivo ocorrer em áreas menores, quando comparado ao cultivo em outros estados e apresentar ausência de padrão, com falhas intratalhões, o que dificulta seu mapeamento.

Mesmo não apresentando expressividade a nível nacional, a atividade sucroalcooleira na região Norte Fluminense-RJ movimenta cerca de 175 milhões de reais a cada safra e emprega, direta e indiretamente, aproximadamente, 15.000 pessoas (AZEVEDO et al. 2002), o que justifica a necessidade de informações atualizadas em apoio ao planejamento da atividade.

Em consonância com o atual estado da arte, este trabalho teve por objetivo realizar, por meio de técnicas de sensoriamento remoto, uma avaliação temporal e quantitativa das áreas ocupadas com a cultura da cana-de-açúcar na região Norte Fluminense - RJ.

\section{MATERIAL E MÉTODO}

\section{Área de estudo}

A área objeto deste trabalho localiza-se às margens do trecho final do Rio Paraíba do Sul, abrangendo os municípios de Campos dos Goytacazes, São Francisco de Itabapoana, São João da Barra, Quissamã, Carapebus e Cardoso Moreira, todos na região norte do Estado do Rio de Janeiro (Figura 1). Segundo LAMEGO (1955), essa região caracteriza-se por uma vasta várzea originária de uma antiga baía que, após regressão marinha e ascensão continental, ocasionou uma planície de grande extensão onde é comum a ocorrência de solos com camadas argilosas, cuja distribuição é aleatória. 
MENDONÇA et al. (2007) citam que a explicação para a distribuição heterogênea dos solos na planície fluvial pode ser a história geológica da região, formada por aportes de sedimentos marinhos e fluviais da era Quaternária, na margem direita, e Terciária, na margem esquerda do Rio Paraíba do Sul. Segundo a classificação climática de Köeppen (1948), a região Norte Fluminense insere-se na classe Aw, isto é, tropical úmido, com verão chuvoso, inverno seco e temperatura do mês mais frio superior a $18{ }^{\circ} \mathrm{C}$. A temperatura média anual situa-se em torno de $24^{\circ} \mathrm{C}$, sendo a amplitude térmica pequena. A precipitação pluviométrica média anual está em torno de $1.020 \mathrm{~mm}$.

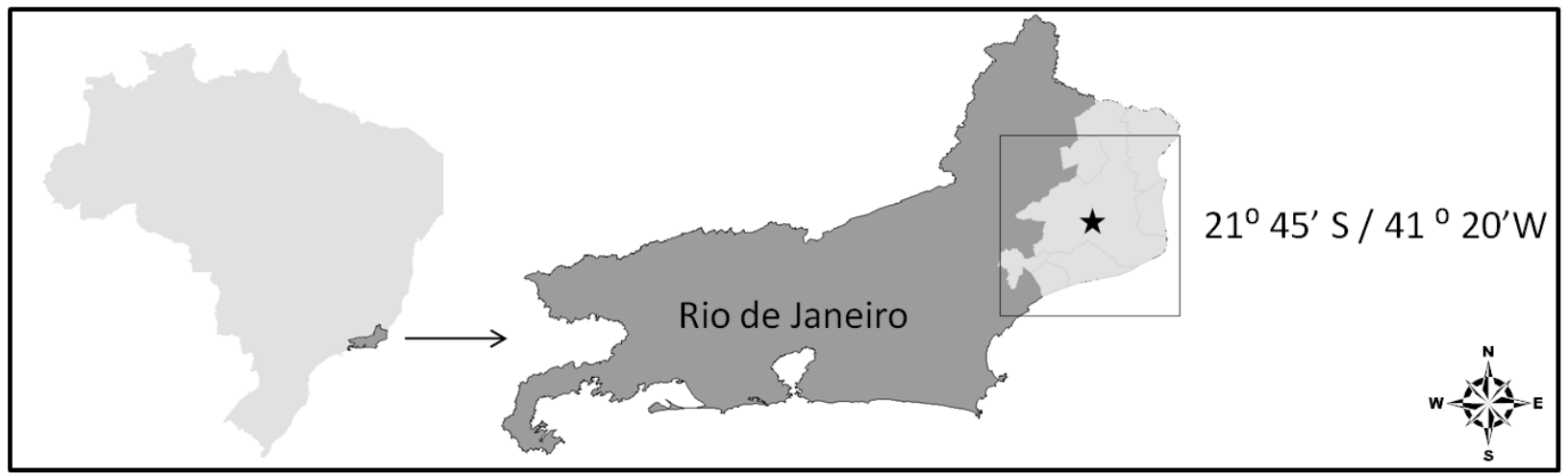

FIGURA 1. Localização da área de estudo. Location of the study area.

\section{Imagens de sensoriamento remoto}

Foram utilizadas imagens adquiridas pelo sensor TM a bordo do satélite Landsat-5. A imagem desse sensor possui resolução espacial de $30 \mathrm{~m}$ x $30 \mathrm{~m}$ (0,09 ha) e resolução temporal de 16 dias. Esse sensor adquire imagens em faixas distintas de comprimento de onda, nas regiões do visível e do infravermelho do espectro eletromagnético. Cada imagem recobre uma área no terreno com dimensão de 185 x $185 \mathrm{~km}$, cuja identificação para fins de localização se dá por meio do número da órbita e do número do ponto dentro de cada órbita. A órbita/ponto da região Norte Fluminense é a 216/75. Sempre que a data e o horário da passagem do satélite, sobre uma determinada área, coincidem com uma atmosfera livre da presença de nuvens, obtém-se uma imagem possível de ser utilizada para a identificação e o mapeamento de alvos da superfície terrestre.

Neste sentido, buscando avaliar a dinâmica espaço-temporal das áreas ocupadas com a cultura da cana-de-açúcar na região, foram selecionadas 18 imagens entre os anos de 1984 a 2007, livres de cobertura de nuvens, correspondentes a seis anos-safra. Cada época foi representada por um mapa de ocupação do solo. Devido à grande variação na resposta espectral da cultura, em função do período amplo de plantio, colheita e tipos de manejo, e visando a aumentar a confiabilidade nos resultados do mapeamento, foi necessário adquirir imagens de períodos diferentes de desenvolvimento. Desta forma, cada mapa é composto de três imagens que caracterizam estádios diferentes de desenvolvimento da cultura. Foram utilizadas as imagens, conforme apresentado na Tabela 1.

TABELA 1. Mapas, datas das imagens Landsat 5 - TM e ano-safra correspondente. Maps, dates of the Landsat 5 - TM images and their corresponding crop years.

\begin{tabular}{ccccc}
\hline Mapa & Data 1 & Data 2 & Data 3 & Ano-Safra \\
\hline 1 & $7-5-1984$ & $23-3-1985$ & $29-7-1985$ & $1984 / 1985$ \\
2 & $17-2-1990$ & $27-7-1990$ & $30-7-1991$ & $1990 / 1991$ \\
3 & $27-1-1994$ & $10-10-1994$ & $7-6-1995$ & $1994 / 1995$ \\
4 & $24-9-2000$ & $15-2-2001$ & $17-7-2001$ & $2000 / 2001$ \\
5 & $1^{\circ}-3-2003$ & $19-10-2003$ & $15-6-2004$ & $2003 / 2004$ \\
6 & $8-8-2006$ & $7-5-2007$ & $28-9-2007$ & $2006 / 2007$ \\
\hline
\end{tabular}




\section{Interpretação das imagens}

Utilizou-se o software SPRING ver. 5.05, obtido gratuitamente no site do Instituto Nacional de Pesquisas Espaciais - INPE. Os mapas contendo a distribuição espacial e temporal das áreas de cana para a região canavieira da região Norte Fluminense foram gerados em duas etapas distintas: interpretação visual na tela do computador e fatiamento da fração vegetação extraída do Modelo Linear de Mistura Espectral (MLME).

\section{Interpretação visual na tela do computador}

Nesta etapa, o objetivo foi mapear as áreas cultivadas com cana-de-açúcar. Para a caracterização do ano-safra, buscou-se utilizar imagens referentes a períodos das fases de desenvolvimento fisiológico, maturação e colheita. Com o intuito de auxiliar no trabalho de interpretação visual, realizou-se a avaliação das três imagens e, dessa forma, uma determinada área que estivesse ocupada com a cultura no período de maturação fisiológica e com solo exposto no período de colheita era editada como "área ocupada pela cultura". Caso, no período de colheita, a área a ser editada apresentasse solo exposto e, no período de perfilhamento e/ou de desenvolvimento fisiológico, apresentasse cana-de-açúcar, a mesma também era editada como "área ocupada pela cultura". A interpretação foi executada de forma interativa até que o intérprete tivesse certeza de que a classe rotulada num dado polígono representasse a realidade no campo.

Além da interpretação visual das imagens, que geraram as "máscaras de cana", aplicou-se o MLME para mapear a "cana em pé" dentro da área mapeada como cana, ou seja, determinando a área da fração vegetação com o objetivo de se estimar a área total ocupada pela cultura.

\section{Modelo Linear de Mistura Espectral (MLME)}

O MLME faz a suposição de que a resposta de um pixel em cada banda espectral é a combinação linear ponderada das respostas espectrais das componentes selecionadas. $\mathrm{O}$ fator de ponderação é dado pela fração da área do pixel ocupada pela respectiva componente (endmember). Para cada pixel individual, o modelo linear de mistura pode ser expresso pela eq.(1):

$$
R_{k}=\sum_{j=1}^{m} r_{j, k} f_{j}+v_{k} \quad(k=1, \ldots, p)
$$

em que,

$\mathrm{R}_{\mathrm{k}}$ - resposta espectral de um pixel na banda espectral k;

$\mathrm{r}_{\mathrm{j}, \mathrm{k}}$ - resposta espectral da componente $\mathrm{j}$ na banda espectral $\mathrm{k}$;

$\mathrm{f}_{\mathrm{j}}$ - fração da área total do pixel ocupada pela componente $\mathrm{j}$;

m - número de componentes;

$\mathrm{v}_{\mathrm{k}}$ - valor do resíduo para a banda espectral k, e

p - número de bandas espectrais em consideração.

As respostas espectrais $R_{k}$ e $r_{j, k}$ podem ser expressas em termos de refletância espectral, radiância espectral ou contador digital. Optou-se por contador digital, uma vez que havia somente o interesse na classificação da imagem.

O MLME foi aplicado nas bandas espectrais dos sensores TM (b1-azul; b2-verde; b3vermelho; b4-infravermelho próximo; b5-infravermelho médio, e b7-infravermelho médio) utilizando três componentes: solo, sombra e vegetação. A fração solo ressalta as áreas de solo exposto; a fração vegetação assemelha-se aos índices de vegetação, ressaltando o vigor vegetativo, e a fração água/sombra ressalta bem os corpos d'água e valores com baixa refletância espectral.

O MLME foi aplicado sobre cada conjunto de imagens ano-safra, e para o cálculo da área foi utilizada a classificação rígida através do fatiamento de histograma. Para esse cálculo, após supervisão do foto-intérprete, considerou-se para fatiamento o limiar conservador para todas as 
imagens de $40 \%$ da imagem fração vegetação, obtendo-se uma imagem binária da área ocupada com a "cana em pé".

\section{RESULTADOS E DISCUSSÃO}

\section{Mapas obtidos pela interpretação visual}

Na Figura 2, são apresentados os mapas das áreas ocupadas com a cultura da cana-de-açúcar nos anos-safra de 1984/1985, 1990/1991, 1994/1995, 2000/2001, 2003/2004 e 2006/2007, gerados pela interpretação visual, que representam as "máscaras de cana".

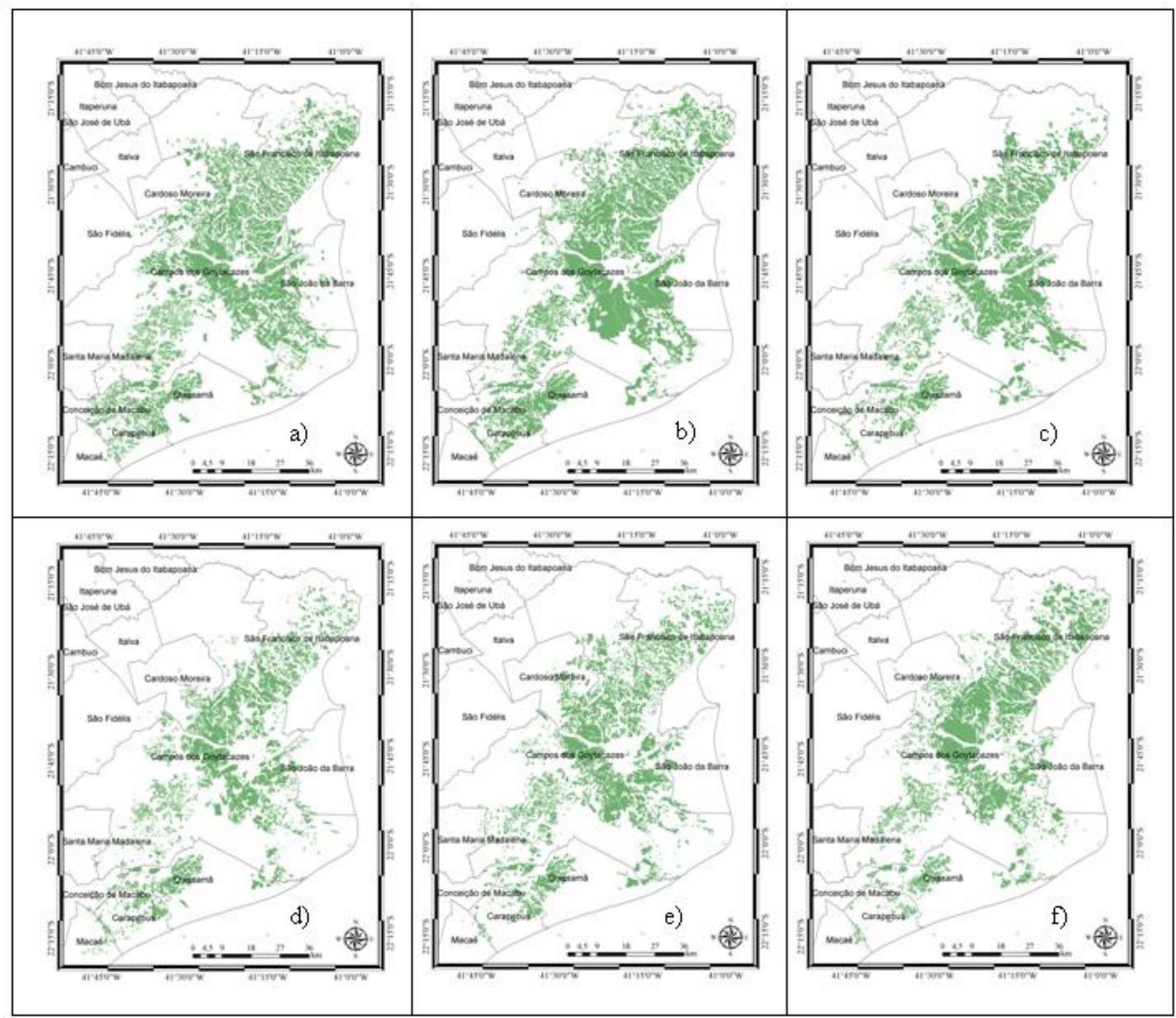

FIGURA 2. Mapas das áreas ocupadas com a cultura da cana-de-açúcar no anos-safra de 1984/1985 (a), 1990/1991 (b), 1994/1995 (c), 2000/2001 (d), 2003/2004 (e) e 2006/2007 (f), gerados pela interpretação visual, Maps of the areas with sugarcane culture of the crop year 1984/85 (a), 1990/91 (b), 1994/95 (c), 2000/01 (d), 2003/04 (e) and 2006/07 (f), generated by the visual interpretation.

\section{Mapas obtidos pelo Modelo Linear de Mistura Espectral (MLME)}

Na Figura 3, são apresentados os mapas das áreas ocupadas com a cultura da cana-de-açúcar nos anos-safra de 1984/1985, 1990/1991, 1994/1995, 2000/2001, 2003/2004 e 2006/2007, gerados pelo Modelo Linear de Mistura Espectral (MLME), que representam a “cana em pé”. 


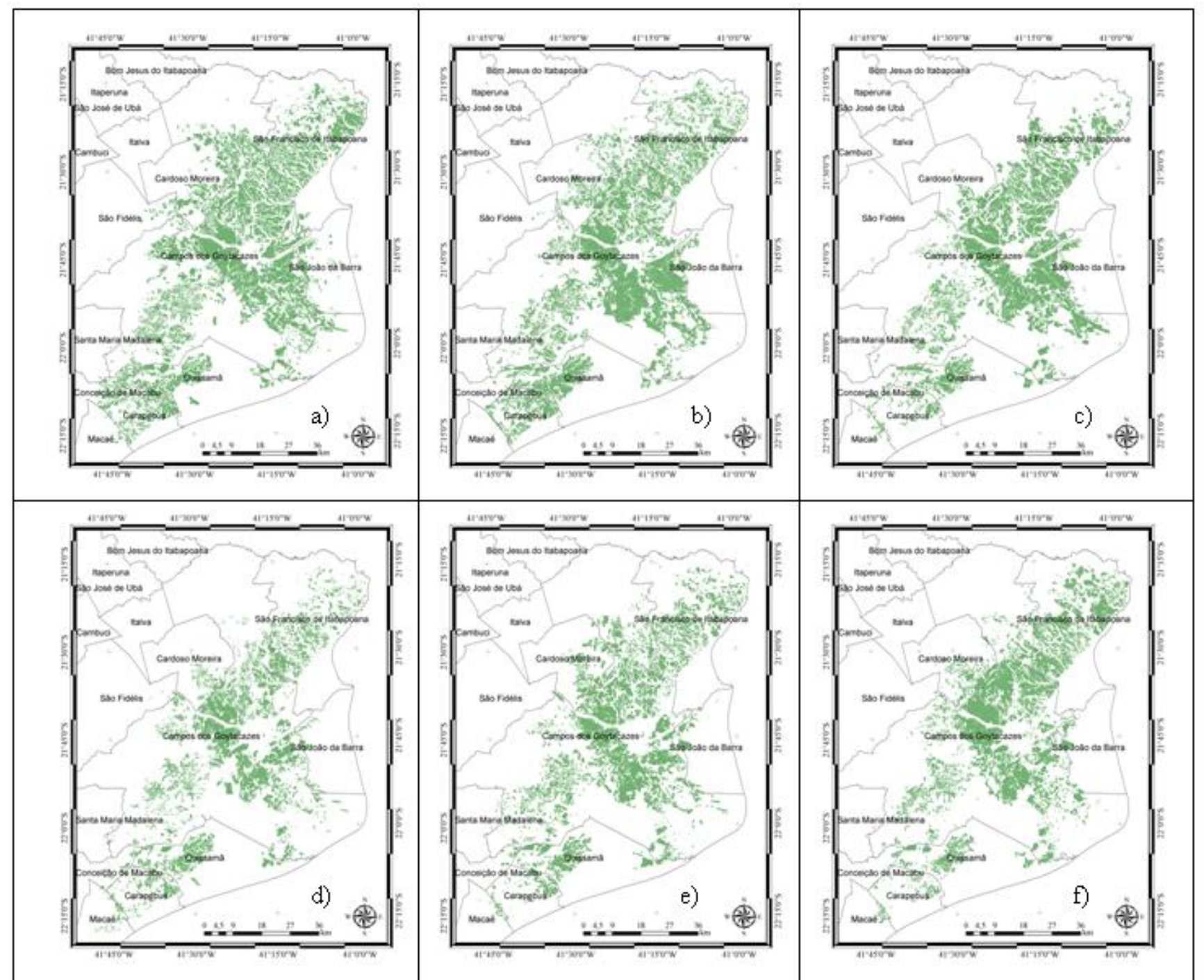

FIGURA 3. Mapas das áreas ocupadas com a cultura da cana-de-açúcar nos anos-safra de 1984/1985 (a), 1990/1991 (b), 1994/1995 (c), 2000/2001 (d), 2003/2004 (e) e 2006/2007 (f), gerados pelo Modelo Linear de Mistura Espectral (MLME). Maps of the areas with sugarcane culture of the crop year 1984/85, 1990/91, 1994/95, 2000/01, 2003/04 and 2006/07, generated by the Spectral Linear Mixing Model (SLMM).

Observando-se as Figuras 2 e 3, pode-se perceber similaridade entre os mapas. No entanto, os mapas da Figura 3 são de coloração mais intensa por excluir as áreas incorporadas como "área de cana" pela interpretação visual. Isso pode ser justificado pelo fato de a técnica do Modelo Linear de Mistura Espectral (MLME) calcular as frações solo, sombra e vegetação para cada pixel da imagem. Assim, os pixels com resposta espectral de outros alvos, que não cana-de-açúcar, terão fração menor de vegetação e, dessa forma, representam melhor a "cana em pé", reduzindo os erros de inclusão de solos expostos por carreadores, falhas nos talhões e valas de drenagem, por exemplo.

$\mathrm{Na}$ Figura 4, são apresentados os mapas das áreas de união (a) das áreas ocupadas com a cultura da cana-de-açúcar durante o período de 1984 a 2007 e intersecção (b) dos mapas dos anos-safra 2006/2007 e 1984/2005 obtido pela aplicação do MLME. 


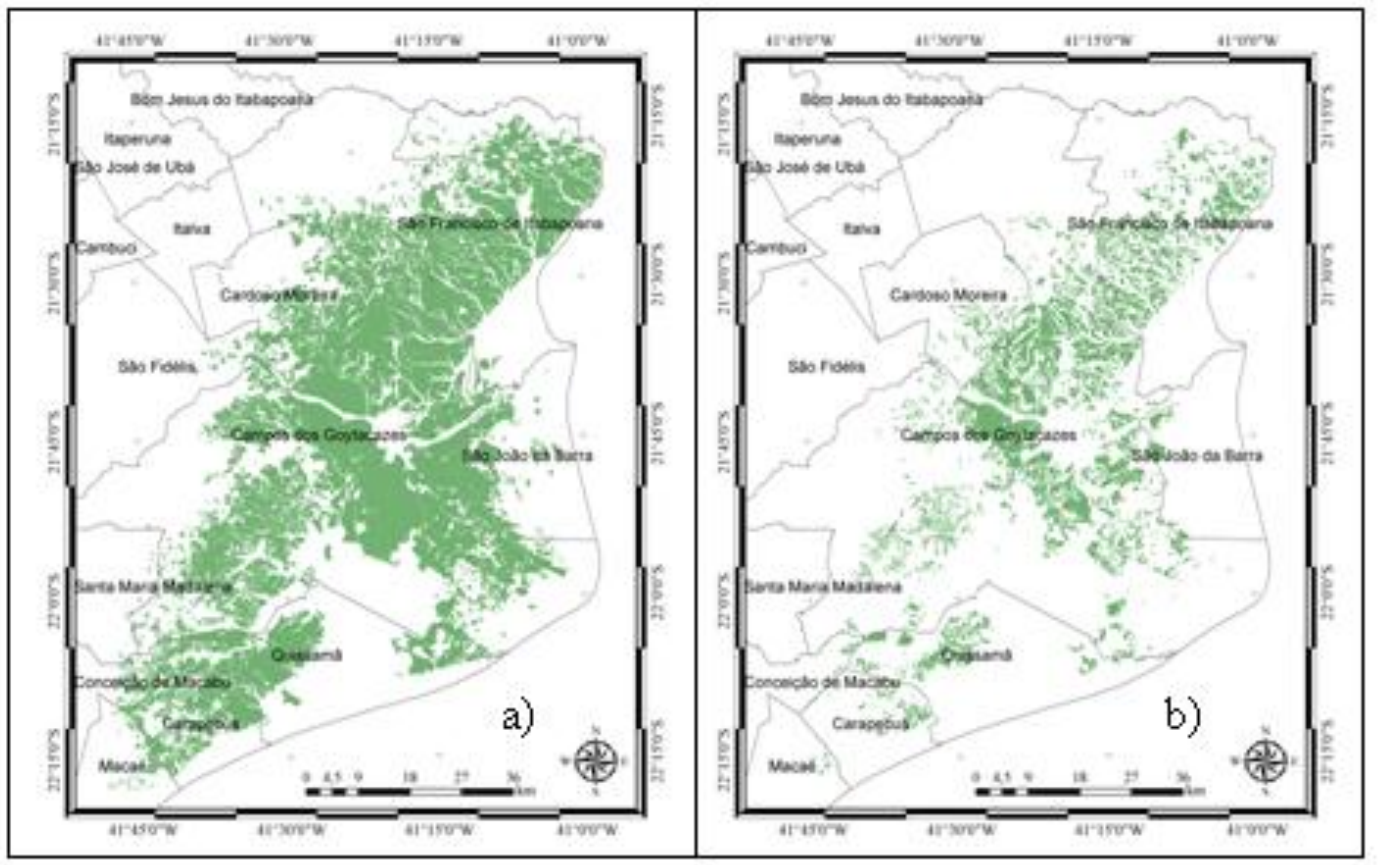

FIGURA 4. Mapas das áreas de união de todos os anos-safra (a) e das áreas de interseção entre os mapas dos anos-safra de 2006/2007 e 1984/2005 (b) ocupadas com cana-de-açúcar na região Norte Fluminense. Maps of the junction (a) and intersection between maps of the areas with sugarcane culture of the crop year 2006/07 and 1984/85 in the North Fluminense Region.

A Figura 4a representa a união de todos os mapas, ou seja, todas as áreas onde, em qualquer ano do período analisado, houve ocupação com a cultura da cana-de-açúcar. A Figura 4.b representa a intersecção entre todos os mapas gerados entre os anos-safra 1996/1997 e 1984/1985, representando as áreas que sempre foram ocupadas pelo cultivo de cana-de-açúcar durante o mesmo período, ou seja, áreas onde sempre ocorreu o uso e a ocupação do solo com a cultura.

A dinâmica espacial do cultivo da cana-de-açúcar no Norte Fluminense - RJ observada na Figura 4 reflete a variação econômica por que passou o setor sucroalcooleiro. Após a extinção do Instituto de Açúcar e Álcool - IAA, em março de 2000, e a perda dos subsídios governamentais, aliados aos baixos preços internacionais do açúcar, a área ocupada com a cultura regrediu drasticamente (OLIVEIRA et al., 2009).

\section{Quantificação das áreas mapeadas}

Nas Tabelas 2 e 3, são apresentadas as áreas, em hectares, ocupadas com a cultura da cana-de-açúcar, obtidas nos mapas gerados pelos métodos de interpretação visual e do Modelo Linear de Mistura Espectral (MLME), respectivamente.

Analisando-se as Tabelas 2 e 3, pode-se perceber que os valores das áreas geradas pelo método do Modelo Linear de Mistura Espectral são inferiores aos valores gerados pelo método da interpretação visual. Essa observação pode ser justificada pelo fato de o MLME representar apenas a "cana em pé", excluindo áreas de estradas e carreadores que refletem a energia emitida pelo solo desnudo, sendo dessa forma mais representativo para a estimativa do uso do solo. 
TABELA 2. Áreas dos municípios do Norte Fluminense ocupadas com cana-de-açúcar nos anossafra 1984/1985, 1990/1991, 1994/1995, 2000/2001, 2003/2004 e 2006/2007, em ha, calculadas pelo método da interpretação visual de Polígonos. Areas in the cities of North Fluminense with sugarcane of the crop years 1984/5, 1990/91, 1994/95, 2000/01, 2003/04 and 2006/07, in ha, calculated by the method of visual interpretation.

\begin{tabular}{|l|c|c|c|c|c|c|}
\hline \multicolumn{1}{|c|}{ Muricipio /Ano-safra } & $\mathbf{1 9 8 4 / 8 5}$ & $\mathbf{1 9 9 0 / 9 1}$ & $\mathbf{1 9 9 4 / 9 5}$ & $\mathbf{2 0 0 0 / 0 1}$ & $\mathbf{2 0 0 3 / 0 4}$ & $\mathbf{2 0 0 6 / 0 7}$ \\
\hline Campos dos Goytacazes & $113.339,42$ & $118.386,25$ & $98.919,54$ & $74.246,31$ & $85.366,34$ & $87.692,86$ \\
\hline S.F. Itabapoana & $30.358,90$ & $37.789,52$ & $23.071,23$ & $18.786,85$ & $21.586,92$ & $31.814,45$ \\
\hline S.J.Barra & $4.631,53$ & $2.885,76$ & $2.788,78$ & $1.556,68$ & $2.448,85$ & $2.235,31$ \\
\hline Quissarnã & $12.298,85$ & $14.889,70$ & $11.521,04$ & $11.048,23$ & $10.437,08$ & $8.677,67$ \\
\hline Cardoso Moreira & $8.952,62$ & $8.693,27$ & $3.747,94$ & $3.151,94$ & $6.460,39$ & $5.753,25$ \\
\hline Carapebus & $9.176,64$ & $10.861,24$ & $3.741,06$ & $5.236,90$ & $3.576,90$ & $2.276,09$ \\
\hline \multicolumn{1}{|c|}{ Total } & $178.757,96$ & $193.505,74$ & $143.789,59$ & $114.026,91$ & $129.876,48$ & $138.449,63$ \\
\hline
\end{tabular}

TABELA 3. Áreas dos municípios do Norte Fluminense ocupadas com cana-de-açúcar nos anossafra 1984/1985, 1990/1991, 1994/1995, 2000/2001, 2003/2004 e 2006/2007, em ha, calculadas pelo Modelo Linear de Mistura Espectral (MLME). Areas in the cities of North Fluminense with sugarcane of the crop years 1984/5, 1990/91, 1994/95, 2000/01, 2003/04 and 2006/07, in ha, calculated by the method of Spectral Linear Mixing Model (MLME).

\begin{tabular}{|c|c|c|c|c|c|c|}
\hline Municipio / Ano-safra & $1984 / 85$ & $1990 / 91$ & $1994 / 95$ & $2000 / 01$ & $2003 / 04$ & $2006 / 07$ \\
\hline Campos dos Goytacazes & $110.706,26$ & $104.133,05$ & $95.953,37$ & $66.286,43$ & $85.079,04$ & $83.578,82$ \\
\hline S.F. Itabapoana & $29.996,15$ & $28.447,84$ & $21.992,16$ & $15.520,15$ & $21.432,03$ & $28.920,49$ \\
\hline S.J.Barra & $4.243,85$ & $2.455,03$ & $2.766,52$ & $1.485,20$ & $2.455,03$ & $2.164,81$ \\
\hline Quissamã & $12.123,08$ & $13.602,51$ & $11.170,79$ & $10.490,62$ & $10.392,58$ & $8.470,63$ \\
\hline Cardoso Moreira & $8.715,93$ & $695.8,38$ & $3.611,26$ & $2.693,10$ & $6.440,09$ & $5.538,57$ \\
\hline Carapebus & $9.024,67$ & $10.100,57$ & $3.554,24$ & $4.814,77$ & $3.574,31$ & $2.146,62$ \\
\hline Total & $174.809,94$ & $158.739,00$ & $139.048,34$ & $101.290,27$ & $129.373,08$ & $130.819,94$ \\
\hline
\end{tabular}

$\mathrm{Na}$ Tabela 4, são apresentadas as áreas ocupadas pela cultura da cana-de-açúcar em cada anosafra de acordo com cada técnica aplicada e os valores percentuais das diferenças.

Analisando a Tabela 4, pode-se perceber que a variação da diferença da área ocupada pela cana-de-açúcar foi sempre menor, em todos os casos, quando obtida pelo MLME, sendo a variação da diferença percentual entre $-0,25$ e $24,72 \%$. A média da diferença percentual entre as duas metodologias foi de $6,02 \%$, com desvio-padrão de $\pm 6,06 \%$ e podem ser justificadas pela existência de carreadores, estradas e valas de drenagem, dentre outros, podendo ser considerados erros de inclusão ao utilizar a metodologia da interpretação visual. Esses resultados obtidos são bastante satisfatórios, uma vez que esses possíveis erros de inclusão estão próximos dos valores comumente relatados na região Norte Fluminense, como sendo em torno de $10 \%$ a $15 \%$ as áreas destinadas à atividade de apoio à produção da cana-de-açúcar. (ASFLUCAN 2010 - comunicado pessoal). 
TABELA 4. Total, por município, das áreas ocupadas com cana-de-açúcar em função da técnica utilizada. Total, by city, of the areas with sugarcane versus the technique applied.

\begin{tabular}{|c|c|c|c|c|c|}
\hline Leal & $\begin{array}{l}\text { Ano } \\
\text { Swin }\end{array}$ & $\begin{array}{c}\text { Mint } \\
\text { Are (b) }\end{array}$ & $\begin{array}{c}\text { IV } \\
\text { Are (b) (a) }\end{array}$ & $\begin{array}{c}\text { DTF } \\
\text { Are (b) }\end{array}$ & $\begin{array}{l}\text { DiF } \\
\text { (6) }\end{array}$ \\
\hline \multirow[t]{6}{*}{ Campos dos Goytacaxes } & 1SATS & 110706,26 & 119999,42 & $-2699,16$ & 2,92 \\
\hline & 199091 & 104.199.ps & 118906,25 & $-14259,20$ & 12,04 \\
\hline & 19945 & 95959,97 & 9019,54 & $-2966,17$ & 9,00 \\
\hline & 200001 & 66206,49 & 74746,91 & -7958 & $\log 72$ \\
\hline & 200904 & s.5079,04 & 5966,94 & $-237,90$ & 094 \\
\hline & 200607 & anstise & 3792,65 & $-4114,04$ & $4, \boldsymbol{\theta}$ \\
\hline \multirow[t]{6}{*}{ SFItbapnana } & 19e4ss & 29996,15 & 9095:so & $-96 ; 5$ & 1,19 \\
\hline & 199091 & 22447,34 & 9770952 & -99416 & 24,72 \\
\hline & 199485 & 21992,16 & 29071,29 & -1079, & 4,6 \\
\hline & 200001 & 15520,15 & 127065 & $-9266, \pi$ & 17,99 \\
\hline & 200904 & 21492,08 & 2156,92 & $-154,9$ & 0,72 \\
\hline & 200607 & 2020,49 & $91 \times 14,45$ & $-2093,6$ & 9,10 \\
\hline \multirow[t]{6}{*}{ S.J. Bann } & 19ans & 4249,5 & 469159 & $-97,6$ & 397 \\
\hline & 199091 & 24559 & 2025,76 & -49073 & 14,99 \\
\hline & 19945 & 2766,52 & $27 a, \pi$ & $-2,26$ & Q 20 \\
\hline & 200001 & 1455,20 & $1556 \%$ & $-\pi, 45$ & 4,9 \\
\hline & 200904 & 245spg & 244585 & 6,18 & $-0,5$ \\
\hline & 200607 & $2164 \$ 1$ & 229591 & $-\pi, 50$ & 9,15 \\
\hline \multirow[t]{6}{*}{ Quissana } & 19ats & 12129,0 & 12025 & $-1 \bar{\pi}, \pi$ & 1,49 \\
\hline & 199091 & 19602,51 & 1420,0 & $-12 \pi, 19$ & 8,64 \\
\hline & 19945 & 111707 & 1152104 & $-9 \sin 25$ & 9,04 \\
\hline & 200001 & 1049062 & 1Lots;y & $-557,61$ & 5,05 \\
\hline & 200904 & 10995 & 10497,0 & $-44,50$ & Q,49 \\
\hline & 200607 & 247069 & 867767 & $-20 /, 04$ & 2,99 \\
\hline \multirow[t]{6}{*}{ Cardinso Mareira } & 19ates & 8715,99 & $\operatorname{ses} 26$ & $-296,9$ & 2,64 \\
\hline & 199091 & 695:ge & 869477 & $-1.794=9$ & 19,86 \\
\hline & 199495 & 9611,36 & $9.747,94$ & $-196,6$ & 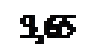 \\
\hline & 200001 & 2694,10 & 9151,94 & $-45 x+4$ & 14,56 \\
\hline & 200904 & 64409 & 6460,9 & -2090 & Q91 \\
\hline & $20060 /$ & $559: 57$ & $5.753,25$ & $-214,6$ & 9,79 \\
\hline \multirow[t]{6}{*}{ Carapebus } & 1SeAts & 90457 & 9176,64 & -15197 & 1,66 \\
\hline & 199091 & $10.100,57$ & $10 \times 61,34$ & $-760,67$ & 7,00 \\
\hline & 199485 & 9554,74 & 9.74106 & $-1 \sin 2$ & 4,99 \\
\hline & 200001 & $4214, \pi$ & 5296,90 & $-42,19$ & 3,06 \\
\hline & 200904 & 9574,91 & 9576,90 & $-2,9$ & qor \\
\hline & 200607 & $2146 \beta$ & $2276 \mathrm{pg}$ & $-129,47$ & $5, \theta$ \\
\hline \multirow[t]{6}{*}{ Área total no ano-sefin } & 19eas & 17420994 & $17 \pi 7,96$ & -9945, & 2,21 \\
\hline & 199091 & 15:79po & 198005,74 & $-94766,74$ & 17,89 \\
\hline & 19945 & 1990:54 & 149759 & $-4741,25$ & 9,90 \\
\hline & 200001 & 10129077 & 11400651 & $-12776,64$ & 11,17 \\
\hline & 200904 & $12997 \mathrm{ps}$ & 129764 & $-50 \mathrm{H}, 40$ & 09 \\
\hline & 200607 & 190519,94 & $19: 449,68$ & -7629 & 5,51 \\
\hline
\end{tabular}

\section{CONCLUSÕES}

Pelos estudos e análises realizados, pode-se concluir que, nos seis principais municípios produtores de cana-de-açúcar localizados na região Norte Fluminense, ocorreu uma queda na área de plantio, durante os anos-safra de 1984/1985 a 2006/2007, de 43.308,33ha (MLME). Pode-se concluir, ainda, que, nos últimos dois anos-safra analisados, houve um incremento na área destinada à atividade canavieira nos municípios da região, principalmente em Campos dos Goytacazes, São 
Francisco de Itabapoana e Cardoso Moreira. Em valores totais, a região recuperou 24.422,72 ha (MLME) entre os anos-safra de 2000/2001 e 2006/2007 e por fim, que a aplicação do método do MLME representa melhor o total da área ocupada pela cultura, uma vez que exclui as áreas de apoio à produção.

\section{AGRADECIMENTOS}

Os autores agradecem ao CNPq (Processo 474827/2006-2) pelo apoio financeiro para a realização desse trabalho.

\section{REFERÊNCIAS}

ASFLUCAN. Associação Fluminense dos Plantadores de Cana. Comunicação pessoal. 2010.

ASSUNÇÃO, G.; DUARTE, V. Avaliação de áreas preparadas para plantio (SOLONU) utilizando dados de satélite Landsat. São José dos Campos: INPE-267-TDL/113, 1983.

ABDEL-RAHMAN, E.M.; AHMED, F.B. The application of remote sensing techniques to sugarcane (Saccharum spp. hybrid) production: a review of the literature. International Journal of Remote Sensing, v.29, n.13, p.3.753-3.767, 2008.

AZEVEDO, H.J.; SILVA NETO, R.; CARVALHO, A.M.; VIANA, J.L.; MANSUR, A.F.U. Uma análise da cadeia produtiva de cana-de-açúcar na região Norte Fluminense. Campos dos Goytacazes: Observatório Sócio-Econômico da Região Norte Fluminense, 2002. 51 p. (Boletim Técnico).

AGUIAR, D.A.; RUDORFF, B.F.; ADAMI, M.; SHIMABUKURO, Y.E. Imagens de sensoriamento remoto no monitoramento da colheita da cana-de-açúcar. Engenharia Agrícola, Jaboticabal, v.29, n.3, p.440-451, 2009.

BENEDIKTSSON, J.A.; SWAIN, P.H.; ERSOY, O.K. Neural network approaches versus statistical methods in classification on multisource remote sensing data. IEEE Tansactions on Geocience and Remote Sensing, New York, v.28, n.4, p.540-522, 1990.

CONAB. COMPANHIA NACIONAL DE ABASTECIMENTO. Disponível em:

<http://www.conab.gov.br/conab web>. Acesso em: 18 set. 2009.

CANASAT (2009). <http://www.dsr.inpe.br/mapdsr/>. Acesso em: 18 set. 2009.

CHEN, S.C.; LIMA, A. M.; MOREIRA, M.A. Estudo comparativo de classificação automática de trigo obtida por vários classificadores. In: SIMPÓSIO BRASILEIRO DE SENSORIAMENTO REMOTO, 2., 1982, Brasília, Anais... p. 951-956.

GOLDEMBERG, J.; GUARDABASSI, P. Are biofuels a feasible option? Energy Policy, Surey, v.37, n.1, p.10-14, 2009.

KÖEPPEN, W. Climatologia: com um estúdio de los climas de la tierra Publications. New Jersey: Climatology. Laboratory of Climatology, 1948. 104 p.

LAMEGO, A.R. Geologia das quadrículas de Campos. São Tomé, Lagoa Feia e Xexé. Rio de Janeiro: DNPM, 1955. 60 p. (Boletim, 154).

MENDONCA, J.C.; FREITAS, R.M.; SOUSA, E.F. Avaliação temporal dos eventos de inundação da Baixada Campista, região Norte Fluminense-RJ, pelo uso de imagens digitais orbitais do sensor Modis. RBC. Revista Brasileira de Cartografia, Rio de janeiro, v.59, n.3, p.249-253, 2007.

OLIVEIRA, A.F.; VICTORIA, D.C.; ANEFALOS, L.C. Monitoramento da safra de cana-de-açúcar: elementos econômicos e contribuições do sensoriamento remoto. In: CONGRESSO DA SOCIEDADE BRASILEIRA DE ECONOMIA, ADMINISTRAÇÃO E SOCIOLOGIA RURAL, 47., 2009, Porto Alegre. 
RUDORFF, B.F.; AGUIAR, D.A.; SILVA, W.F. Studies on the rapid expansion of sugarcane for ethanol production in São Paulo State (Brazil) using Landsat data. Remote Sensing, Ottawa, v. 2, n.4, p.1.057-1.076, 2010. doi: 10.3390/rs2041057

SABOL, D.E.; GILLESPIE, A.R.; ADMNS, J.B.; SMITH, M.O.; TUCKER, C.J. Structural stage in Pacific Northwest estimated using simple mixing models of multispectral images. Remote Sensing of Environment, Ottawa, n.80, p.1-16, 2002.

SHIMABUKURO, Y.E.; BATISTA, G.T.; MELLO, E.M.K.; MOREIRA, J.C.; DUARTE, V. Using shade fraction image segmentation deforestation in Landsat Thematic Mapper images of the Amazon Region. International Journal of Remote Sensing, v.19, n.3, p.535-541, 1998.

SHIMABUKURO, Y.E.; SMITH, J.A. The least-squares mixing models to generate fraction images derived from remote sensing multispectral data. IEEE Transactions on Geoscience and Remote Sensing, New York, v.29, p.16-20, 1991. 